\title{
Development and Validation of Computational Models for Mammalian Circadian Oscillators
}

\author{
DANIEL B. FORGER, ${ }^{1}$ DENNIS A. DEAN II, ${ }^{2}$ KATHERINE GURDZIEL, \\ JEAN-CHRISTOPHE LELOUP, ${ }^{3}$ CHOOGON LEE, ${ }^{4}$ CHARLOTTE VON GALL, ${ }^{4}$ \\ JEAN-PIERRE ETCHEGARAY, ${ }^{4}$ RICHARD E. KRONAUER, ${ }^{2}$ \\ ALBERT GOLDBETER, ${ }^{3}$ CHARLES S. PESKIN, ${ }^{1,5}$ MEGAN E. JEWETT, \\ and DAVID R. WEAVER ${ }^{4}$
}

\begin{abstract}
Circadian rhythms are endogenous rhythms with a cycle length of approximately $24 \mathrm{~h}$. Rhythmic production of specific proteins within pacemaker structures is the basis for these physiological and behavioral rhythms. Prior work on mathematical modeling of molecular circadian oscillators has focused on the fruit fly, Drosophila melanogaster. Recently, great advances have been made in our understanding of the molecular basis of circadian rhythms in mammals. Mathematical models of the mammalian circadian oscillator are needed to piece together diverse data, predict experimental results, and help us understand the clock as a whole. Our objectives are to develop mathematical models of the mammalian circadian oscillator, generate and test predictions from these models, gather information on the parameters needed for model development, integrate the molecular model with an existing model of the influence of light and rhythmicity on human performance, and make models available in BioSpice so that they can be easily used by the general community. Two new mammalian models have been developed, and experimental data are summarized. These studies have the potential to lead to new strategies for resetting the circadian clock. Manipulations of the circadian clock can be used to optimize performance by promoting alertness and physiological synchronization.
\end{abstract}

\section{INTRODUCTION}

$\mathbf{T}$ The Mammalian CiRCADian TIMING System is responsible for the regulation of approximately 24-h rhythmicity in many physiological functions. The objective of this effort is to understand this system biologically and to model it mathematically, leading to the generation of experimentally testable hypotheses

\footnotetext{
${ }^{1}$ Courant Institute, New York University, New York, New York.

${ }^{2}$ Biomathematical Modeling Unit, Division of Sleep Medicine, Brigham \& Women's Hospital and Harvard Medical School, Boston, Massachusetts.

${ }^{3}$ Unité de Chronobiologique Théorique, Université Libre de Bruxelles, Brussels, Belgium.

${ }^{4}$ Department of Neurobiology, University of Massachusetts Medical School, Worcester, Massachusetts.

${ }^{5}$ Center for Neural Science, New York University, New York, New York.
} 
and the potential for development of strategies to manipulate the circadian pacemaker. Our work focuses on the 24-h biochemical oscillations that occur within individual cells. The suprachiasmatic nuclei (SCN) of the anterior hypothalamus contain the master circadian pacemaker in mammals. Long-term recordings of electrical activity rhythms from SCN neurons indicate that individual cells can maintain circadian timekeeping (Herzog et al., 1998; Liu et al., 1997; Welsh et al., 1995). Furthermore, mutations that influence rhythmicity in the whole animal have a corresponding effect on individual "clock cells" in vitro (Herzog et al., 1998; Liu et al., 1997). Thus, in terms of fundamental brain mechanisms, the circadian system is among the most tractable models for providing a complete understanding of the cellular and molecular events connecting genes to behavior. A second focus of investigation is identification of the biological mechanisms by which light influences the mammalian circadian clock, and incorporation of the influence of light into our mathematic models. We also intend to compare the influence of light in the molecular model with our existing model of the influence of light and rhythmicity on human performance.

\section{Basics of circadian rhythms}

Numerous aspects of physiology and behavior vary over the course of the $24-\mathrm{h}$ day. The most widely appreciated daily rhythm is the sleep-wake cycle. Fluctuations in alertness, sleep latency, and other functions occur with a cycle length of approximately $24 \mathrm{~h}$. These fluctuations persist even in the absence of sleep, or in experimental protocols where sleep is allowed but is distributed evenly (in brief segments) over the 24-h cycle.

A second, widely appreciated aspect of rhythmicity is revealed by the phenomenon called "jet lag." After flight across several time zones, there is often a period of several days during which the individual is "out of synch" with their environment. During this period, there is a discrepancy between the body's biological time and the actual local time. A period of several days of re-adjustment follows, characterized by altered sleep-wake cycles and numerous (but minor) physiological and psychological complaints. After several days in the new environment, the body becomes resynchronized to the local environment. Unfortunately, this often occurs just in time for the return trip home.

The body "knows what time it is" as a result of a biological timekeeping system. This system can be thought of as consisting of a pacemaker (or clock), along with the input pathways that allow information to reach it, and the output pathways that lead to overt rhythms in physiology and behavior (Fig. 1). A critical defining feature of circadian rhythms is that rhythmicity persists with a cycle length of approximately ( irca) one day (diem, thus the term circadian), even in constant environmental conditions. A second feature is that the clock mechanism can be reset by environmental stimuli. Light is the most effective agent for synchronizing the circadian clock to the 24-h day in most species.

While rhythms in physiology and behavior are often studied at the whole-organism or tissue level, the underlying circadian oscillatory machinery can reside within individual cells. Indeed, circadian rhythms have been described in unicellular organisms, including cyanobacteria (Dunlap et al., 1999) and in individual neurons isolated from the mammalian SCN (Herzog et al., 1998; Liu et al., 1997; Welsh et al., 1995). While the master clock regulating behavior is located in the SCN of the anterior hypothalamus (Reppert and Weaver, 2002), there are also circadian oscillators in other tissues (Abe et al., 2002; Balsalobre et al., 1998; Reppert and Weaver, 2002; Yagita et al., 2000; Yamazaki et al., 2000; Zylka et al., 1998). The same genes involved in the intracellular SCN clock mechanism are rhythmically expressed in other brain areas and in peripheral organs, both in vivo and in vitro. These extra-SCN oscillators apparently can sustain 24-h oscillations for only a few days without input from the SCN, while SCN oscillations persist for weeks to months in cells and tissues in vitro (Abe et al., 2002; Balsalobre et al., 1998; Yamazaki et al., 2000). The SCN synchronizes the timing of extra-SCN ("peripheral" or "slave") oscillators (for review, see Schibler et al., 2003). Synchronized slave oscillators, in turn, regulate local (tissue-specific) rhythms in physiology and behavior. From an experimental viewpoint, the existence of peripheral oscillators allows us to characterize molecular rhythms in normal and mutant mice using peripheral tissues (e.g., liver, skeletal muscle, and cell lines) that are much more abundant than the very small SCN. We have made extensive use of peripheral tissues as a surrogate for the SCN, with the belief that the molecular components of the intracellular feedback loops are similar across tissues. 


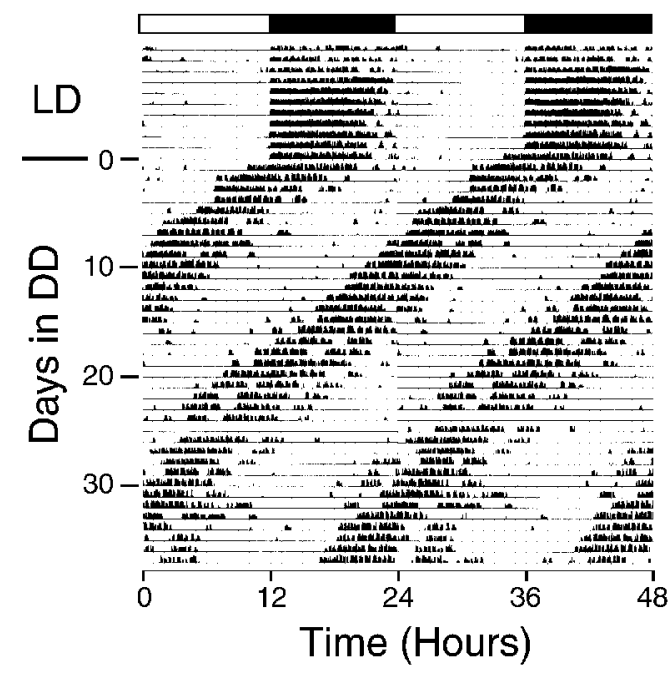

FIG. 1. Critical features of circadian rhythms, illustrated by mouse locomotor activity. Activity of a mouse in a wheel in its cage is represented by vertical deflections (bars) on each horizontal line. The horizontal lines represent $48 \mathrm{~h}$ of continuous recording; data are "double-plotted," with day 2 of data being shown both to the right and below day 1. Note that running wheel activity occurs at night in mice housed in a light-dark cycle (LD, light cycle shown across the top of the panel, with light period indicated by the open portion of the bar). When the animal is placed into constant darkness (DD), it continues to express rhythmicity of behavior, but with a cycle length of less than $24 \mathrm{~h}$. Thus, rhythms are regulated by an endogenous system and are not simply a passive response to environment. However, light is a critical stimulus for synchronizing the endogenous rhythmicity to environmental conditions. The circadian timing system regulates rhythmicity in many aspects of physiology and behavior. (Modified from Bae et al., 2001.)

\section{Molecular basis of the mammalian circadian clock}

At the molecular level, circadian oscillations are based on the rhythmic expression of clock genes. The intracellular clock mechanism in the mouse $\mathrm{SCN}$ is based primarily on a transcriptional-translational negative feedback loop (Figs. 2 and 3). Molecular elements that comprise this system in mammals have been identified recently (Fig. 3). Two basic helix-loop-helix (bHLH)/PAS-containing transcription factors, CLOCK and BMAL1 (MOP3) provide the basic drive to the system by activating transcription of negative regulators through E box enhancer elements (Bunger et al., 2000; Gekakis et al., 1998; Hogenesch et al., 1998; Jin et al., 1999; King et al., 1997; Vitaterna et al., 1994). CLOCK:BMAL1 heterodimers drive transcription of three Period genes (mPerl-3) and two Cryptochrome genes (mCryl-2) (Reppert and Weaver, 2002). mPER and mCRY proteins form complexes, translocate to the nucleus, and interact with CLOCK:BMAL1 heterodimers to inhibit transcription, closing the feedback loop (Kume et al., 1999; Lee et al., 2001; van der Horst et al., 1999; Vitaterna et al., 1999).

Analysis of mice with targeted disruption of the circadian-relevant genes described above reveals the importance of these genes in circadian rhythmicity (Fig. 3; for review, see Reppert and Weaver 2002). Mutations leading to transcriptionally non-functional CLOCK or BMAL1 leads to loss of rhythmicity in constant darkness. While the Drosophila cryptochrome homolog functions as a circadian photoreceptor, mouse mCRY1 and mCRY2 are redundant, and are essential components of the negative limb of the clock feedback loop (van der Horst et al., 1999; Vitaterna et al., 1999). Mice deficient in either mPER1 or mPER2 have altered period length and delayed loss of rhythmicity following transfer to constant darkness, while mice lacking both mPER1 and mPER2 show a more severe phenotype, immediate arrhythmicity on placement in constant conditions (Bae et al., 2001; Zheng et al., 2001). mPER3 does not have a critical role in the maintenance of the core clock feedback loops (Bae et al., 2001; Shearman et al., 2000a). 


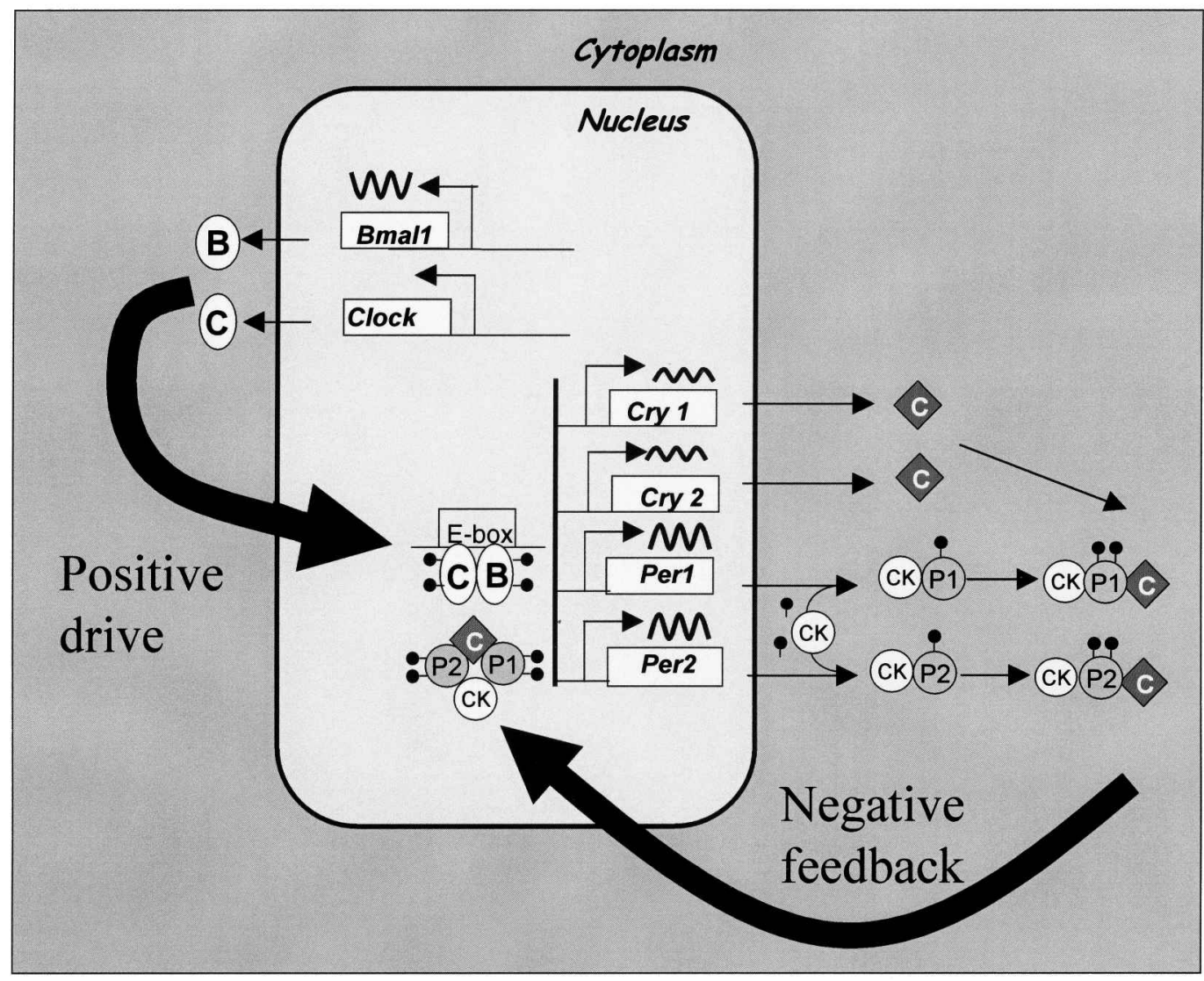

FIG. 2. Schematic representation of the intracellular circadian clock in mammals. CLOCK and BMAL1 dimerize and drive transcription of $m P e r 1, m P e r 2, m C r y 1$, and $m C r y 2$ through E box elements. These transcripts are then translated to proteins that are phosphorylated (by casein kinase), dimerize, and enter the nucleus. These large multiprotein complexes then interact with promoter-bound CLOCK:BMAL1 heterodimers to turn off transcription. A feedback loop involving the rhythmic regulation of Bmall, through activity of the repressor, REVERB-alpha, is not shown. (Adapted from Reppert and Weaver, 2002.)

A "positive loop" regulates the rhythmic expression of Bmall (Preitner et al., 2002; Shearman et al., 2000b). Bmall RNA levels are rhythmic, and the rhythms are in antiphase to those for the $m P e r$ and $m C r y$ genes. Rhythmic Bmall transcription is due to rhythmic repression by REVERB-alpha (Preitner et al., 2002; Ueda et al., 2002). RevErb-alpha RNA is regulated through E box elements, and has a phase of production similar to that of mPers and mCrys. mPER2 may also play a role in stimulating Bmall expression (Shearman et al., 2000b; Zheng et al., 1999). This positive feedback loop regulating the expression of Bmall appears to be less important than the negative feedback loop in the mammalian system. Studies of REVERBalpha-deficient mice reveal that Bmall RNA levels in the SCN are constantly high and not rhythmic, consistent with the proposal that rhythmically produced REVERB-alpha normally acts to repress Bmall transcription (Preitner et al., 2002). Nevertheless, REVERB-alpha-deficient mice maintain circadian locomotor activity rhythms in constant conditions, indicating that rhythmic Bmall expression is not necessary for circadian clock function. We have extended this observation in several ways.

\section{Models of molecular circadian oscillators}

Previous work on mathematical modeling of molecular circadian oscillators has focused on Drosophila (Goldbeter, 1995; Leloup and Goldbeter, 1998, 1999; Smolen et al., 2001, 2002; Tyson et al., 1999; Ueda et al., 2001). From a biological perspective, homologs of most of the genes involved in the mammalian cir- 


\section{Clock Protein Families}

Protein Features

\begin{tabular}{ll|l|l|l|}
\hline & & \\
Q-rich \\
MOPOC
\end{tabular}

PER1

PER2

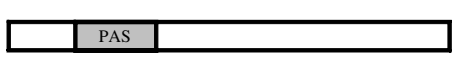

PER3

CRY1

CRY2

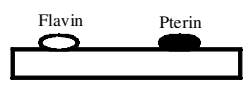

$\mathrm{CKI} \varepsilon$

CKI $\delta$
Clock Gene Mutations

\begin{tabular}{|c|c|c|}
\hline Gene & Type & Behavioral Phenoty \\
\hline Clock & Deletion & Long period to arrhythmic \\
\hline$B M A L 1$ & Null & Arrhythmic \\
\hline Perl & Null & Var. period to arrhythmic \\
\hline Per2 & Null & Var. period to arrhythmic \\
\hline Per3 & Null & Short period \\
\hline Perl/2 & Null/Null & Arrhythmic \\
\hline Cryl & Null & Short period \\
\hline Cry2 & Null & Long period \\
\hline Cryl/2 & Null/Null & Arrhythmic \\
\hline CKIE & Missense & Short period ( $\operatorname{tau}$ hamster) \\
\hline$C K I \delta$ & Not Done & Unknown \\
\hline
\end{tabular}

FIG. 3. Molecular components of the mammalian circadian clockwork. (Left) Four families of proteins are known to be important for circadian rhythms in mammals. Within the basic helix-loop-helix-PAS family, CLOCK and BMAL1 are most important for behavioral rhythms, while their close homologs, MOP4 (also called NPAS2) and MOP9 (also called BMAL2) appear less important within the central clock. The Period gene family has three members, $m$ Perl, $m P e r 2$, and $m P e r 3$. The Cryptochrome family has two members, $m C r y 1$ and $m C r y 2$. Two casein kinases, CK1 epsilon and CK1 delta, are capable of phosphorylating PER proteins as well as other circadian proteins. Signature structural domains for each gene family are indicated. (Right) Mutations alter circadian behavioral rhythms in mice. For the $P e$ riod and Crytochrome gene families, partial redundancy of function is apparent. No mutations of CK1 delta have been reported. (Adapted from Reppert and Weaver, 2002.)

cadian clockwork were first identified in the fly circadian clock, and the general core clock mechanism of interacting transcriptional feedback loops is similar between flies and mice (Reppert and Weaver, 2000; Young and Kay, 2001). There has been a shuffling of functions between the components, however, and gene duplication in mammals has increased the complexity of the mammalian feedback loops. Nevertheless, interaction between groups studying Drosophila and mammalian oscillators, or the mathematical models that describe them, provide fertile grounds for information transfer. Within the BioCOMP program, the Byrne group is working on Drosophila circadian models. Mathematical models of the Drosophila oscillator have provided the starting point for one mammalian model, developed with partial support from the BioCOMP program (Leloup and Goldbeter, 2003). A second, more detailed, strictly mammalian model of the mammalian circadian oscillator has been developed based, to the extent possible, on experimental data collected as a part of this research program (Forger and Peskin, 2003).

\section{METHODS AND RESULTS}

\section{Experimental group cumulative results}

Posttranslational mechanisms in the circadian clock. While the critical structure of the circadian feedback loop is known to be a transcriptional-translational feedback loop, little was known regarding the pro- 
teins and their post-transcriptional modifications. Efforts to characterize circadian protein rhythms have focused on liver tissue, and have required generation of specific antisera for mouse proteins (Lee et al., 2001). These antisera have been used for three types of experiments: Western blot analysis (detection of protein levels and molecular size), immunoprecipitation (isolation of protein complexes by precipitation with an antibody, then identification of co-precipitating proteins by Western blot analysis), and chromatin immunoprecipitation (isolation of chromatin fragments by precipitation with an antibody to protein bound to DNA, followed by assessment of the levels of specific DNA sequences in the precipitated material).

The analysis of circadian protein rhythms revealed several important findings (Lee et al., 2001). Absolute levels of the mCRY proteins are significantly higher than PER levels. Cellular fractionation studies reveal that mCRY is present in the cytoplasm throughout the circadian cycle. Nuclear entry of the mCRY and mPER proteins occurs simultaneously, both in liver and in SCN (Hastings et al., 1999; Lee et al., 2001), suggesting that the highly rhythmic production of mPER proteins is the key to regulating nuclear entry of the complex consisting of the negative regulators (Lee et al., 2001).

An unexpected finding was that the levels of casein kinase I epsilon exceed PER levels throughout the circadian cycle (Lee et al., 2001). This situation indicates that the enzyme is actually more abundant than its substrate.

Consistent with previous work in Drosophila, analysis of mouse proteins revealed temporal changes in phosphorylation state of circadian proteins, especially mPER1, mPER2, CLOCK, and BMAL1 (Lee et al., 2001). These changes in phosphorylation state (detected by alterations in electrophoretic mobility) occur coincident with changes in cellular localization and inter-molecular interactions, indicating an important role for post-translational mechanisms in clock function.

Details of transcriptional mechanisms. The accessibility of DNA for interaction with transcription factors is strongly influenced by the interaction of DNA and histone proteins in nucleosomes. Post-translational alterations in histone $\mathrm{H} 3$ and histone $\mathrm{H} 4$ (e.g., phosphorylation, acetylation, and methylation) at specific residues in the amino-terminal tail of these proteins are highly correlated with alterations in transcriptional activity of specific genes. In liver, a circadian rhythm in histone modification occurs at the promoters of the mPerl, mPer 2 , and $m$ Cryl genes (Etchegaray et al., 2003). The acetylation of histone $\mathrm{H} 3$ is rhythmic, and the rhythm parallels the rhythms in mPerl RNA levels and in RNA polymerase II binding to the promoter. The rhythmic acetylation of histone $\mathrm{H} 3$ is likely accompanied by other covalent modifications (e.g., histone phosphorylation and methylation) that collectively alter chromatin structure. Thus, the circadian rhythmicity in transcription appears to be regulated by a rhythm in chromatin remodeling which prepares the promoter region for the activation/inhibition cycle. The $\mathrm{mCRY}$ proteins may repress CLOCK:BMAL1-mediated transcription by inducing alterations in chromatin structure (e.g., disrupting a coactivator complex or recruitment of a histone deacetylase activity).

Mechanisms of circadian entrainment in the SCN. A critical feature of the circadian timing system is the ability to be entrained by the environmental light/dark cycle. Time-dependent responsiveness to light is a characteristic feature of circadian clocks in diverse species (Dunlap, 1999). Exposure of mice to light early in the night shifts the clock so that subsequent cycles begin at a later time. Exposure to light late in the night, in contrast, advances the circadian clock.

Several lines of evidence suggest that mPER 1 and mPER2 play important roles in mediating phase-shifting responses to light (for review, see Reppert and Weaver, 2002; Bae and Weaver, 2003). Our studies of mice with disruption of $m P e r$ genes indicate, however, that neither mPER1 nor mPER2 is absolutely required for phase-shifting by light (Bae and Weaver, 2003). This suggests that multiple molecular pathways may be activated in response to light, and that effort should be spent on identifying these pathways. Recently, induction of DEC gene expression in the SCN in response to light has been reported (Honma et al., 2002); the functional importance of this event also should be investigated.

One alternative pathway for light-induced resetting of the circadian clock has been proposed involving rapid, light-induced destruction of BMAL1 protein. (This would be an attractive mechanism, as light-induced degradation of the TIM protein is critical for resetting of the Drosophila circadian pacemaker.) This proposal is based on immunoblot studies conducted with rat SCN. The studies revealed a circadian rhythm 
in BMAL1 protein, with peak levels occurring at night, and rapid degradation of BMAL1 after exposure to light at night (Tamaru et al., 2000). We have recently reexamined this issue in mice. Our study (von Gall et al., 2003) shows that BMAL1 and CLOCK proteins are continuously expressed at high levels in the mouse SCN, supporting the hypothesis that rhythmic negative feedback plays the major role in rhythm generation in the mammalian pacemaker. Furthermore, light exposure did not lead to degradation of BMAL1 protein in the mouse $\mathrm{SCN}$, as assessed by both immunocytochemistry and immunoblot analysis (von Gall et al., 2003). These results indicate that rapid degradation of BMAL1 protein is not a consistent feature of resetting mechanisms in rodents.

Experimental group work in progress. Many of our conclusions regarding the relative amounts of circadian proteins and their interactions are derived from studies in liver (Lee et al., 2001). We are working on confirming these findings in other tissues using methods similar to those described previously. Determination of the accuracy of the parameter values from liver in at least one additional tissue is an important, but time-consuming, effort.

We continue to work with several cell lines with the objective of being able to induce rhythmicity under controlled conditions and also to visualize rhythmicity within individual cells. Specifically, we have generated a number of constructs with destabilized fluorescent reporter proteins under control of E-box containing promoter elements that should oscillate in vitro after experimental perturbation (e.g., serum shock; see Balsalobre et al., 1998; Yagita et al., 2001). However, we have had poor luck to date in generating stable cell lines that show the expected rhythmic expression patterns. We also have worked rather extensively with stable human cell lines with inducible expression of constructs that direct expression of mouse circadian proteins (O. Gildemeister, K. Bae, O. Froy, and D.R. Weaver, unpublished data). Additional characterization of the cell lines is continuing, and we are testing additional manipulations for ability to induce rhythmicity. Detection of rhythmicity in individual cells will allow us to gather important information on the precision of oscillations within individual pacemakers, revealing both the cycle-to-cycle variability as well as the variation between cells. Almost all existing data addresses the mean behavior of populations of oscillators without addressing the behavior of individual oscillators. Analysis of oscillations in single cells will impact our understanding of oscillator robustness and the importance of molecular noise. Our future plans are to continue the work in progress as described above, and to test model predictions generated by the Modeling Group regarding molecular shuttling and the influence of protein:protein interactions on protein localization and stability. We will also investigate mechanisms for cooperativity at $\mathrm{E}$ box elements in the promoter of responsive genes.

\section{Modeling group cumulative results}

Two quite distinct molecular models of the mammalian circadian oscillator have been developed. There are several features that one would expect to be achieved by a circadian model (Table 1) and both models meet the major criteria. Within each model, there are terms for the processes influencing each molecular entity (Fig. 4), including RNA production (transcription) and degradation, intracellular movement of RNA, protein and protein/protein complexes, interactions between proteins and alteration in their localization, stability, and activity as a result of these interactions. The major difference between the two models is the level of biochemical detail which they represent.

A mammalian model developed as an adaptation of a Drosophila model. Jean-Christophe Leloup and Albert Goldbeter have developed a deterministic model of the mammalian circadian pacemaker (Leloup and Goldbeter, 2003). The modeling techniques used are similar to a previous model they developed of the Drosophila circadian oscillator (Leloup and Goldbeter, 1998). The Leloup-Goldbeter (2003) mammalian model does not distinguish among the products of the three $m P e r$ genes or the two $m C r y$ genes, and instead uses single $m P e r$ and $m C r y$ entities (RNA and proteins), and with phosphorylation as a mechanism controlling protein degradation. Enzyme-substrate interactions are modeled with Michaelis-Menten type expressions, and transcription regulation is modeled by Hill-type expressions. The model consists of 16 equations. By the addition of three additional equations and the modification of one other, the model can be 
Table 1. Draft Criteria for Assessing Circadian Oscillatory Models

Criterion

1. Period

2. Phase

3. Entrainment

4. Phase response

5. Mutations
Expected model performance

Molecular oscillations occur with a free-running period (tau) of approximately $24 \mathrm{~h}$.

(a) Molecular oscillations occur with appropriate phase relationships to each other in freerunning conditions (appropriate $=$ consistent with experimental data or plausible).

(b) Molecular oscillations occur with appropriate phase relationships to each other and to the light-dark cycle.

(a) Input repeated at 24-h intervals results in 24-h periodicity of the molecular oscillations.

(b) The molecular basis for the input to influence molecular oscillations should be based on experimental data.

(a) Single stimuli lead to alterations in the phase of molecular oscillation.

(b) The response to a stimulus depends on the phase at which it is administered.

(c) The molecular basis for the input to influence molecular oscillations should be based on experimental data.

Mutations affecting the level or activity of circadian-relevant genes in vivo should produce similar effects on oscillations in silico.

modified to include the influence of REV-ERB-alpha on Bmall transcription. Light input to the model is achieved through elevation of $\mathrm{mPer}$ levels. Two benefits of this approach are the limited number of equations and that the model can also be used as a Drosophila model simply by renaming variables and changing the effects of light.

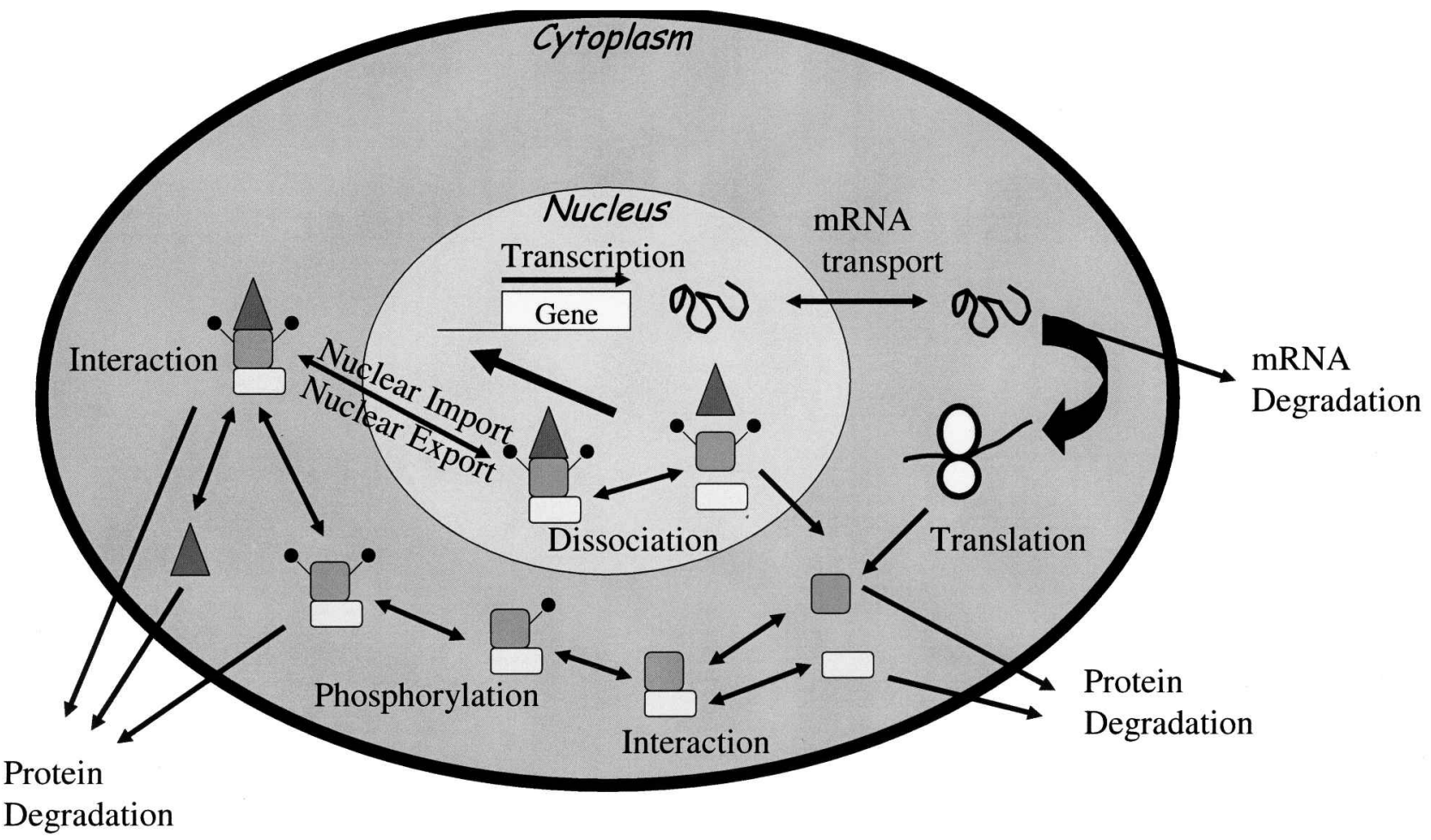

FIG. 4. Biochemical processes considered in mammalian model. Processes included in models of the circadian oscillator are RNA transcription, transport and degradation, protein production, protein modification (by phosphorylation), and interactions, degradation, and entry of multimeric complexes into the nucleus. This schematic represents steps for mPER1 protein (square), including interaction with casein kinase (rectangle), leading to phosphorylation (addition of small black "lollipops"), and interaction with mCRY proteins (triangle). 
Development of a mammalian model based on experimental data. Recent data on the mammalian circadian oscillator provide a detailed view of transcription regulation, as well as post-translational events, including phosphorylation. To model these data, the actual binding between kinases and substrates must be used instead of Michaelis-Menten dynamics. In addition, data from the experimental group provides a more detailed picture of transcription regulation than Hill-type expressions allows. A new mammalian model (Forger and Peskin, 2003) has been developed which considers these details. The model incorporates a series of 73 equations. The molecular entities tracked in the model include: mPER1, mPER2, mCRY1, mCRY2, and casein kinase I proteins, and their corresponding mRNAs. CLOCK and BMAL1 are constitutively expressed. PER3 is not included in the model, as it appears not to play a role in the core circadian feedback loop (Bae et al., 2001; Shearman et al., 2000a). Where indicated by experimental data, the model considers multiple phosphorylation states of several of these proteins. Incorporating this level of detail allows us to test the differential roles of mPER1 and mPER2 in phase resetting, simulate mutations in individual proteins (e.g., mPER1 or mPER2), and study specific aspects of phosphorylation (e.g., the tau mutation) or transcription regulation. This model achieves a good agreement with experimental data (Fig. 5).

Modeling group future directions. The major objectives of the Modeling Group in the future will be development of a stochastic version of the Forger-Peskin mammalian model, sensitivity analysis of the model, and mathematical reduction of the model to allow its integration as an input component to an existing model of the human circadian pacemaker and its role in regulating neurobehavioral performance. More specifically, a model exists describing the influence of light on the human circadian pacemaker and on performance (Forger et al., 1999; Jewett and Kronauer, 1998, 1999; Jewett et al., 1999; Kronauer et al., 1999). In a previous modeling and analytical study, Forger and Kronauer (2002) showed how a biochemical circadian clock model (Goldbeter, 1995) had essentially the same behavior as a model describing the influence of light on the human circadian pacemaker (Forger et al., 1999). In line with this study, our project objective is to develop and simplify, then merge the detailed molecular models with this existing performance model. To do this, we will need to conduct extensive analyses to determine the most critical variables to consider in the simplified format. This analysis is ongoing. Our Software Group will participate

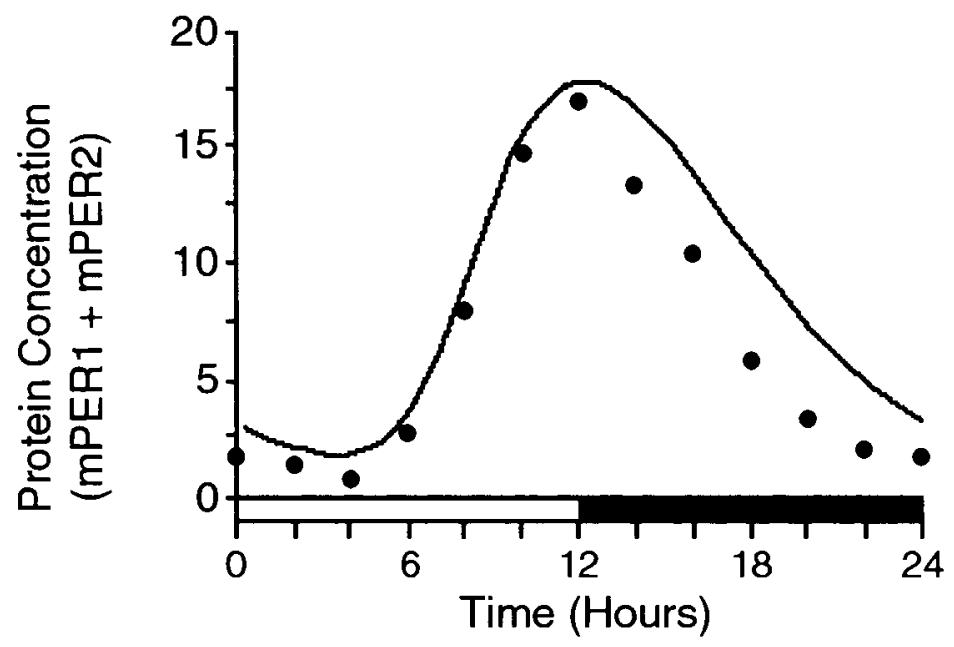

FIG. 5. Comparison of model predictions with experimental data. Experimental data (points) for the time profile of total mPER levels were generated from data on the mPER1 and mPER2 rhythms in whole cell extracts from mouse liver, determined by quantitative western blotting (Lee et al., 2001). The model predictions are from the mammalian model developed by D.B. Forger and C. Peskin (2003). Shown is model prediction (line) and experimental data (points) in darkness after entrainment to a lighting cycle consisting of 12-h light and 12-h darkness per 24 h. The amplitude and shape of the profile are correctly predicted, and the phase of entrainment of the model accurately reflects the experimental data. 
through their knowledge of BioSpice tools that may be useful in simplifying the model structure. Additional areas that we intend to pursue in the future include comparison of the two mammalian models, investigation of model behavior in the presence or absence of the REV-ERB-alpha loop, and a more detailed investigation of entrainment mechanisms.

\section{Software group cumulative results}

Software submissions in support of our BioCOMP program effort provide resources to allow the BioSpice community, and eventually the public, to work with published circadian oscillator models. The models currently available through the BioCOMP website are listed in Table 2. Models have been submitted in several formats, consistent with the moving target for development of models and their distribution. Most recently, we have translated three models into SBML as part of our support for BioSPICE agents, in order to allow the agents to pass Experimental Working Group data types or SBML structures between the facilitators or agents. We used both Jigcell and Cellerator (www.aig.jpl.nasa.gov/public/mls/cellerator/) to create SBML-compatible implementations of three published Drosophila models (Goldbeter, 1995; Leloup and Goldbeter, 1998; Ueda et al., 2001).

Current software efforts are focused on assisting model development and in preparing the newly developed mammalian models for submission in BioSpice-compatible format. We are also involved in development of user interfaces for the models (Fig. 6).

Our future plans are to (a) continue to submit molecular models to the BioCOMP program according to the current state of the art, and (b) investigate resources available within the BioCOMP program, (c) use these resources to promote modeling efforts, and (d) interact effectively with other groups and developers to identify areas where additional work is needed. As we begin integration of the molecular model with the human performance model, a significant effort will be devoted to identification of BioCOMP resources that will facilitate model development. Areas of major importance include automatic parameter fitting and identifying tools for determining the quality of fit for objective comparison of model predictions with experimental results.

The Software Group (Dennis Dean and Katherine Gurdziel) will remain instrumental in our efforts to make circadian oscillatory models available in a BioSpice environment and in a user-friendly format. This will include providing examples of the user interfaces and also gathering feedback from biologists on the "accessibility" of BioCOMP program resources.

\section{DISCUSSION}

The circadian oscillator represents a useful test case for the principle underlying the BioCOMP program. A relatively circumscribed set of molecular and genetic interactions leads to a biological event, oscillation, with a period of approximately $24 \mathrm{~h}$. The oscillations of individual cells are representative of oscillatory mechanisms that occur at the organismal level. Furthermore, these oscillations have important functional

Table 2. Circadian Models in the NeuroRhythms Area of the Sandbox

\begin{tabular}{llcccc}
\hline Model & Species & Matlab & SBML & Parameters & Documentation \\
\hline Goldbeter, 1995 & Drosophila & + & + & + & + \\
Leloup and Goldbeter, 1998 & Drosophila & + & + & + & + \\
Ueda et al., 2001 & Drosophila & + & + & + & + \\
Leloup and Goldbeter, 1999 & Drosophila & + & + & ++ & + \\
Leloup and Goldbeter, 2003 & Mammalian & + & + & + & + \\
Forger and Peskin, 2003 & Mammalian & + & + & + & + \\
\hline
\end{tabular}

+ , Information currently posted to the NeuroRhythms section of the BioSpice website.

++ , Both Chaos and Birhythmicity parameter sets are included. 

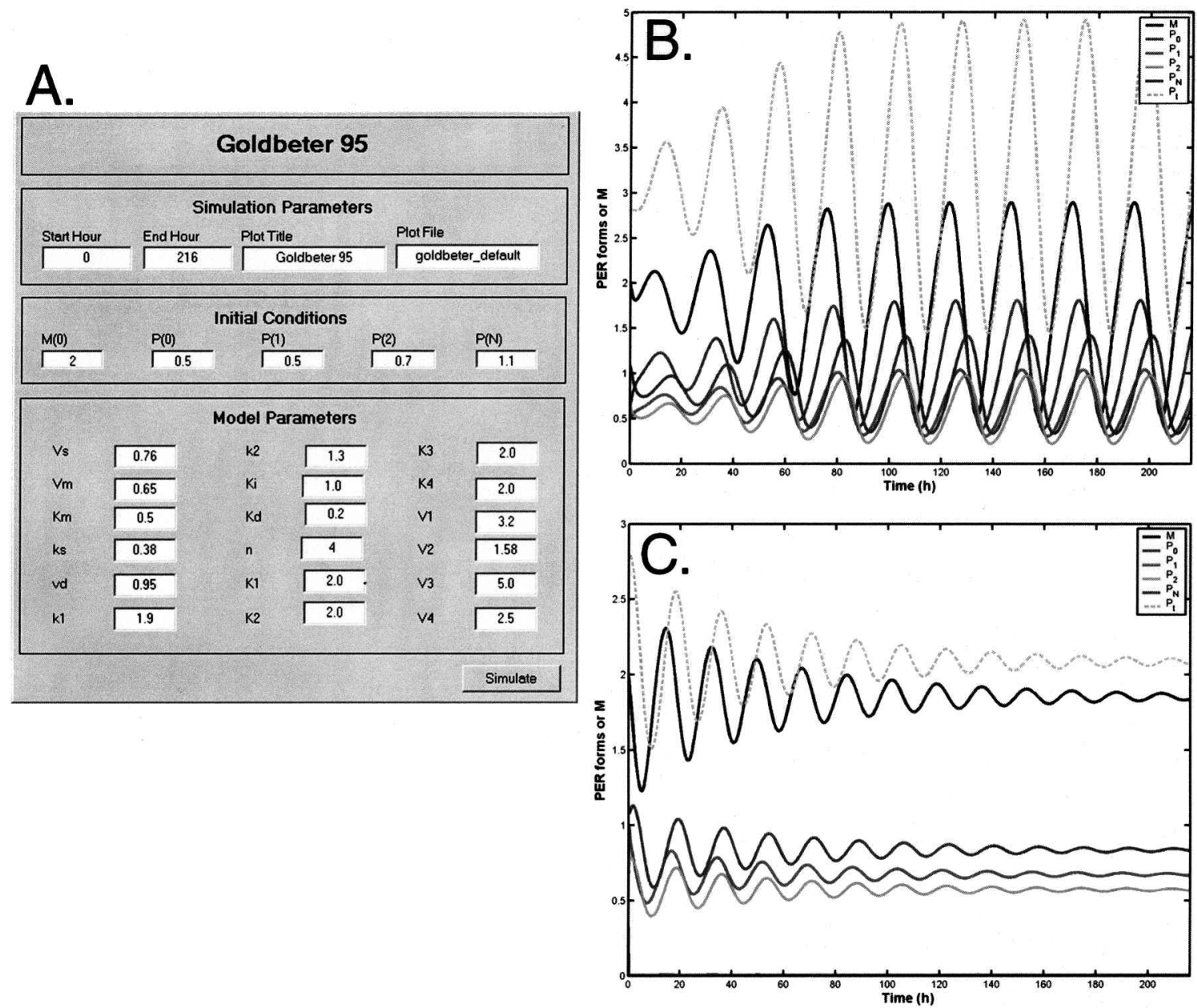

FIG. 6. Representative circadian model user interface created in Matlab. Representative "screenshots" depicting the User Interface for the Goldbeter (1995) Drosophila model. (A) Parameter input screen. (B) Output screen. Robust circadian rhythmicity in the abundance of the molecular species is present despite a slight change from the default parameter set. The only change from default is that $\mathrm{k}_{1}$, the transfer rate of PER into the cytoplasm, has been changed from the default value of 2.0 to 1.9. (C) The amplitude of rhythmicity is greatly reduced within several cycles when $\mathrm{K}_{1}$, the Michaelis constant of PER in the cytoplasm, is changed from 2.0 to 0.01 . The user interface and models are accessible in the Jewett Team submissions in the NeuroRhythms section of the Sandbox of the BioSPICE website (www.biospice.org). (From D. Dean and K. Gurdziel, unpublished.)

implications. Optimization of work schedules through attention to circadian principles is now more common in the workplace, and the use of timed light exposure to optimize performance and mission readiness offers significant advantages over current pharmacological approaches, such as the use of alertness-promoting stimulants. Thus, we have a cellular model system for experimental analysis and mathematical modeling, with tangible implications for human performance.

Parallel development of mammalian models by our research team and of Drosophila models by the Byrne group (Smolen et al., 2001, 2002) within the BioCOMP Program provides important opportunities for interaction and exploration of the specific aspects of model behavior. The group of investigators led by Jim Schwaber focuses on gene regulatory networks and signal transduction events, particularly those occurring after angiotensin receptor activation, a stimulus that can initiate rhythmicity in cultured cells (Nonaka et al., 2001). Environmental light information is conveyed to the SCN oscillator by neurochemical pathways, 
leading to activation of signal transduction pathways that convey input from the cell surface into the oscillator, and leading to transcriptional responses. Thus, there is likely overlap in the cellular mechanisms occurring in our experimental models. Furthermore, the Schwaber group has worked with mathematical models of circadian rhythms (Zak et al., 2001). Finally, the Tyson group also has experience with modeling oscillators, including circadian oscillators (Tyson et al., 1999). These groups participate in the NeuroRhythms group, which can be found in the Sandbox on the BioSpice website (www.BioSpice.org), and would be a good starting point for those seeking additional information about recent advances in this area of research.

Our long-term objective is to achieve a comprehensive understanding of the circadian oscillator itself, as well as how light exposure impacts the oscillator. This information should allow identification of novel pharmacological approaches to either stop the clock or reset it with precision, regardless of ambient lighting conditions. Control over the circadian oscillator should promote adaptation to shift work and non24-h duty cycles, and minimize the physiological disturbance caused by jet lag.

\section{ACKNOWLEDGMENTS}

This effort was sponsored by the Defense Advanced Research Projects Agency (DARPA) and the Air Force Research Laboratory under agreement number F30602-01-2-0554. The U.S. Government is authorized to reproduce and distribute reprints for Government purposes notwithstanding any copyright annotation thereon. D.B. Forger was supported by an NSF pre-doctoral fellowship. J.-C. Leloup is Chargé de recherches du Fonds National de la Recherche Scientifique (F.N.R.S., Belgium). Portions of the Experimental Group work reported here were also supported by NIH grant NS39303 to S.M. Reppert and the Emmy-Noether Programm of the Deutsche Forschungsgemeinschaft to C. von Gall. The contents of this publication are solely the responsibility of the authors and do not necessarily represent the official views of the awarding agencies.

\section{REFERENCES}

ABE, M., HERZOG, E.D., YAMAZAKI, S., et al. (2002). Circadian rhythms in isolated brain regions. J. Neurosci. 22, $350-356$.

BAE, K., and WEAVER, D.R. (2003). Light-induced phase shifts in mice lacking mPER1 or mPER2. J. Biol. Rhythms 16, 123-133.

BAE, K., JIN, X., MAYWOOD, E.S., et al. (2001). Differential functions of $m P e r 1, m P e r 2$, and $m P e r 3$ in the SCN circadian clock. Neuron 30, 525-536.

BALSALOBRE, A., DAMIOLA, F., and SCHIBLER, U. (1998). Circadian rhythms in isolated brain regions. Cell 93, 929-937.

BUNGER, M.K., WILSBACHER, L.D., MORAN, S.M., et al. (2000). Mop3 is an essential component of the master circadian pacemaker in mammals. Cell 103, 1009-1017.

DUNLAP, J.C. (1999). Molecular bases for circadian clocks. Cell 96, 271- 290.

ETCHEGARAY, J.P., LEE, C., WADE, P.A., et al. (2003). Rhythmic histone acetylation underlies transcription in the mammalian circadian clock. Nature 421, 177-182.

FIELD, M.D., MAYWOOD, E.S., O'BRIEN, J.A., et al. (2000). Analysis of clock proteins in mouse SCN demonstrates phylogenetic divergence of the circadian clockwork and resetting mechanisms. Neuron 25, $437-447$.

FORGER, D.B., and PESKIN, C.S. (2003). A detailed predictive model of the mammalian circadian clock. Proc. Nat'l. Acad. Sci. USA (in press).

FORGER, D.B., JEWETT, M.E., and KRONAUER, R.E. (1999). A simpler model of the human circadian pacemaker. J. Biol. Rhythms 14, 532-537.

FORGER, D.B., and KRONAUER, R.E. (2002). Reconciling mathematical models of biological clocks by averaging on approximate manifolds. SIAM J. Appl. Math. 62, 1281.

GEKAKIS, N., STAKNIS, D., NGUYEN, H.B., et al. (1998). Role of the CLOCK protein in the mammalian circadian mechanism. Science 280, 1564-1569. 


\section{COMPUTATIONAL MODELS FOR MAMMALIAN CIRCADIAN OSCILLATORS}

GOLDBETER, A. (1995). A model for circadian oscillations in the Drosophila period protein (PER). Proc. R. Soc. Lond. B Biol. Sci. 261, 319-324.

HASTINGS, M.H., FIELD, M.D., MAYWOOD, E.S., et al. (1999). Differential regulation of mPER1 and mTIM proteins in the mouse suprachiasmatic nuclei: new insights into a core clock mechanism. J. Neurosci. 19, RC11.

HERZOG, E.D., TAKAHASHI, J.S., and BLOCK, G.D. (1998). Clock controls circadian period in isolated suprachiasmatic nucleus neurons. Nat. Neurosci. 1, 708-713.

HOGENESCH, J.B., GU, Y.-Z., JAIN, S., et al. (1998). The basic-helix-loop-helix-PAS orphan MOP3 forms transcriptionally active complexes with circadian and hypoxia factors. Proc. Natl. Acad. Sci. USA 95, 5474-5479.

HONMA, S., KAWAMOTO, T., TAKAGI, Y., et al. (2002). Dec1 and Dec2 are regulators of the mammalian molecular clock. Nature 419, 841-844.

JEWETT, M.E., and KRONAUER, R.E. (1998). Refinement of a limit cycle oscillator model of the effects of light on the human circadian pacemaker. J. Theor. Biol. 192, 455-465.

JEWETT, M.E., and KRONAUER, R.E. (1999). Interactive mathematical models of subjective alertness and cognitive throughput in humans. J. Biol. Rhythms 14, 588-597.

JEWETT, M.E., FORGER, D.B., and KRONAUER, R.E. (1999). Revised limit cycle oscillator model of human circadian pacemaker. J. Biol. Rhythms 14, 493-499.

JIN, X., SHEARMAN, L.P., WEAVER, D.R., et al. (1999). A molecular mechanism regulating rhythmic output from the suprachiasmatic circadian clock. Cell 96, 57-68.

KING, D.P., ZHAO, Y., SANGORAM, A.M., et al. (1997). Positional cloning of the mouse circadian Clock gene. Cell 89, 641-653.

KUME, K., ZYLKA, M. J., SRIRAM, S., et al. (1999). mCRY1 and mCRY2 are essential components of the negative limb of the circadian clock feedback loop. Cell 98, 193-205.

KRONAUER, R.E., FORGER, D.B., and JEWETT, M.E. (1999). Quantifying human circadian pacemaker response to brief, extended, and repeated light stimuli over the phototopic range. J. Biol. Rhythms 14, 500-515.

LEE, C., ETCHEGARAY, J.P., CAGAMPANG, F.R., et al. (2001). Posttranslational mechanisms regulate the mammalian circadian clock. Cell 107, 855-867.

LELOUP, J.-C., and GOLDBETER, A. (1998). A model for circadian rhythms in Drosophila incorporating the formation of a complex between the PER and TIM proteins. J. Biol. Rhythms 13, 70-87.

LELOUP, J.-C., and GOLDBETER, A. (1999). Chaos and birhythmicity in a model for circadian oscillations of the PER and TIM proteins in Drosophila. J. Theor. Biol. 198, 445-459.

LELOUP, J.-C., and GOLDBETER, A. (2003). Toward a detailed computational model for the mammalian circadian clock. Proc. Natl. Acad. Sci. USA 100, 7051-7056.

LIU, C., WEAVER, D.R., STROGATZ, S.H., et al. (1997). Cellular construction of a circadian clock: period determination in the suprachiasmatic nuclei. Cell 91, 855-860.

NONAKA, H., EMOTO, N., IKEDA, K., et al. (2001). Angiotensin II induces circadian gene expression of clock genes in cultured vascular smooth muscle cells. Circulation 104, 1746-1748.

PREITNER, N., DAMIOLA, F., LOPEZ-MOLINA, L., et al. (2002). The orphan nuclear receptor REV-ERB-alpha controls circadian transcription within the positive limb of the mammalian circadian oscillator. Cell 110, 251-260.

REPPERT, S.M., and WEAVER, D.R. (2000). Comparing clockworks: mouse versus fly. J. Biol. Rhythms 15, $357-364$.

REPPERT, S.M., and WEAVER, D.R. (2002). Molecular bases for circadian clocks. Nature 418, 935-941.

SCHIBLER, U., RIPPERGER J., and BROWN, S.A. (2003). Peripheral circadian rhythms in mammals: time and food. J. Biol. Rhythms 18, 250-260.

SHEARMAN, L.P., JIN, X., LEE, C., et al. (2000a). Targeted disruption of the mPer3 gene: subtle effects on circadian clock function. Mol. Cell. Biol. 20, 6269-6275.

SHEARMAN, L.P., SRIRAM, S., WEAVER, D.R., et al. (2000b). Interacting molecular loops in the mammalian circadian clock. Science 288, 1013-1019.

SMOLEN, P., BAXTER, D.A., and BYRNE, J.H. (2002). A reduced model clarifies the role of feedback loops and time delays in the Drosophila circadian oscillator. Biophys. J. 83, 2349-2359.

SMOLEN, P., BAXTER, D.A., and BYRNE, J.H. (2001). Modeling circadian oscillations with interlocking positive and negative feedback loops. J. Neurosci. 21, 6644-6656.

TAMARU, T., ISOJIMA, Y., YAMADA, T., et al. (2000). Light- and glutamate-induced degradation of the circadian oscillating protein BMAL1 during the mammalian clock resetting. J. Neurosci. 20, 7525-7530.

TYSON, J.J., HONG, C.I., THRON C.D., et al. (1999). A simple model of circadian rhythms based on dimerization and proteolysis of PER and TIM. Biophys. J. 77, 2411-2417.

UEDA, H. R., CHEN, W., ADACHI, A., et al. (2002). A transcription factor response element for gene expression during circadian night. Nature 418, 534-539. 
UEDA, H.R., HAGIWARA, M., and KITANO, H. (2001). Robust oscillations within the interlocked feedback model of Drosophila circadian rhythm. J. Theor. Biol. 210, 401-406.

van DER HORST, G.T., MUIJTJENS, M., KOBAYASHI, K., et al. (1999). Mammalian Cry1 and Cry2 are essential for maintenance of circadian rhythms. Nature 398, 627-630.

VITATERNA, M.H., KING, D.P., CHANG, A.M., et al. (1994). Mutagenesis and mapping of a mouse gene, Clock, essential for circadian behavior. Science 264, 719-725.

VITATERNA, M.H., SELBY, C.P., TODO, T., et al. (1999). Differential regulation of mammalian period genes and circadian rhythmicity by cryptochromes 1 and 2. Proc. Natl. Acad. Sci. USA 96, 12114-12119.

VON GALL, C., NOTON, E., LEE, C., et al. (2003). Light does not degrade the constitutively expressed BMAL1 protein in the mouse suprachiasmatic nucleus. Eur. J. Neurosci. 18, 125-133.

WELSH, D.K., LOGOTHETIS, D.E., MEISTER, M., et al. (1995). Individual neurons dissociated from rat suprachiasmatic nucleus express independently phased circadian firing rhythms. Neuron 14, 697-706.

YAGITA, K., TAMANINI, F., vAN DER HORST, G.T., et al. (2001). Molecular mechanisms of the biological clock in cultured fibroblasts. Science 292, 278.

YAMAZAKI, S., NUMANO, R., ABE, M., et al. (2000). Resetting central and peripheral circadian oscillators in transgenic rats. Science 288, 682-685.

YOUNG, M.W., and KAY, S.A. (2001). Time zones: a comparative genetics of circadian clocks. Nat. Rev. Genet. 2, 702-715.

ZAK, D.E., DOYLE, F.J., III, VLACHOS, D.G., et al. (2001). Stochastic kinetic analysis of transcriptional feedback models for circadian rhythms. Presented at the 40th IEEE Conference on Decision and Control.

ZHENG, B., ALBRECHT, U., KAASIK, K., et al. (2001). Nonredundant roles of the mPerl and mPer 2 genes in the mammalian circadian clock. Cell 105, 683-694.

ZHENG, B., LARKIN, D.W., ALBRECHT, U., et al. (1999). The mPer2 gene encodes a functional component of the mammalian circadian clock. Nature 400, 169-173.

ZYLKA, M.J., SHEARMAN, L.P., WEAVER, D.R., et al. (1998). Three Period homologs in mammals: differential light responses in the suprachiasmatic circadian clock and oscillating transcripts outside of brain. Neuron 20, $1103-1110$.

Address reprint requests to:

David R. Weaver, Ph.D.

Department of Neurobiology

Lazare Research Building, Room 723

University of Massachusetts Medical School

364 Plantation St.

Worcester, MA 01605-2324

E-mail: David.Weaver@umassmed.edu 